\title{
Crystal structure of anhydrous tetraethylammonium chloride, $\left[\left(\mathrm{CH}_{3} \mathrm{CH}_{2}\right)_{4} \mathbf{N}\right] \mathrm{Cl}$
}

\author{
R. J. Staples* \\ Harvard University, Department of Chemistry and Chemical Biology, Cambridge, MA 02138, USA
}

Received November 16, 1998, CCDC-No. 1267/60

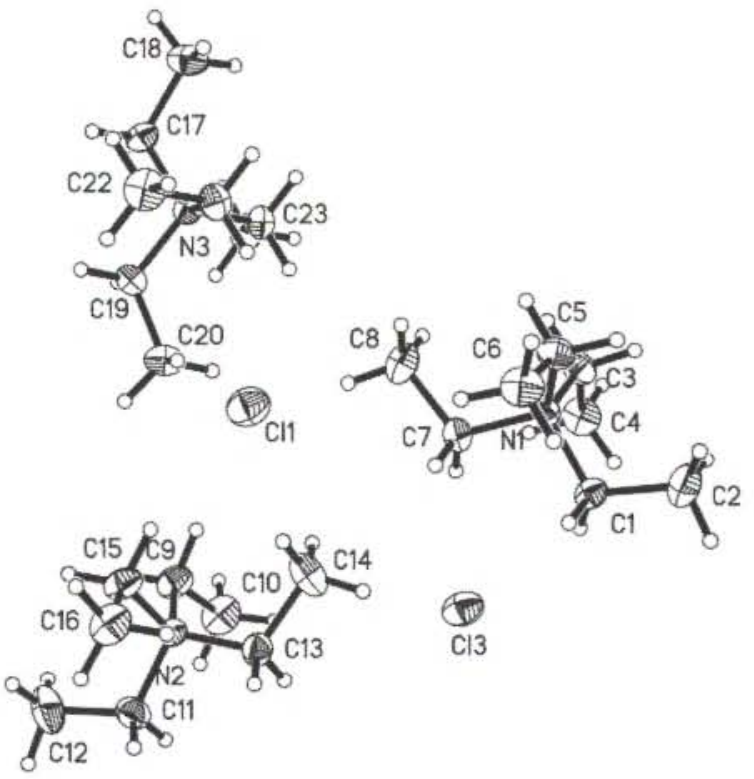

\begin{abstract}
$\mathrm{C}_{8} \mathrm{H}_{20} \mathrm{ClN}$, monoclinic, $P 121 / n 1$ (No. 14), $a=11.8207(2) \AA$, $b=20.4092(4) \AA, c=12.4290(1) \AA, \beta=108.173(3)^{\circ}$, $V=2849.0 \AA^{3}, Z=12, R_{\mathrm{gt}}(\mathrm{F})=0.053, R_{\mathrm{w}}\left(F^{2}\right)=0.116$, $T=213 \mathrm{~K}$.
\end{abstract}

\section{Source of material}

The compound was isolated from acetonitrile as a side product. The crystals were grown in anhydrous acetonitrile in a dry atmosphere.

\section{Discussion}

The structure is the similar to those reported previously, although there are three molecules per assymmetric cell and there are no waters of hydration. The structure reported by Loechlin and Kvick [1] is the monohydrate and Mak, Bruins, Slot and Beurskens [2] reported the tetrahydrate structure.

\footnotetext{
* Correspondence author (e-mail: staples@chemistry.harvard.edu)
}

Table 1. Data collection and handling.

$\begin{array}{ll}\text { Crystal: } & \text { colorless plate, size } 0.05 \times 0.10 \times 0.15 \mathrm{~mm} \\ \text { Wavelength: } & \text { Mo } K_{\alpha} \text { radiation }(0.71073 \AA) \\ \mu: & 3.38 \mathrm{~cm}^{-1} \\ \text { Diffractometer, scan mode: } & \text { SMART CCD, } \omega, \delta \omega=0.3^{\circ} \\ 2 \theta_{\text {max: }} & 56.76^{\circ} \\ N(h k l)_{\text {measured }}, N(h k l)_{\text {unique: }} & 18492,6893 \\ \text { Criterion for } I_{\text {obs, }} N(h k l)_{\mathrm{gt}}: & I_{\mathrm{obs}}>2 \sigma\left(I_{\mathrm{obs}}\right), 5145 \\ N(\text { param })_{\text {refined: }} & 266 \\ \text { Programs: } & \text { SHELXS-97 [3], SHELXL-97 [4] }\end{array}$

Table 2. Atomic coordinates and displacement parameters (in $\AA^{2}$ ).

\begin{tabular}{|c|c|c|c|c|c|}
\hline Atom & Site & $x$ & $y$ & $z$ & $U_{\text {iso }}$ \\
\hline$N(3)$ & $4 e$ & $0.4582(1)$ & $-0.26034(7)$ & $0.2334(1)$ & $0.0225(3)$ \\
\hline $\mathrm{H}(1 \mathrm{~A})$ & $4 e$ & 0.3901 & 0.1511 & 0.1940 & 0.034 \\
\hline $\mathrm{H}(1 \mathrm{~B})$ & $4 e$ & 0.4251 & 0.1485 & 0.3276 & 0.034 \\
\hline $\mathrm{H}(2 \mathrm{~A})$ & $4 e$ & 0.4965 & 0.2445 & 0.2720 & 0.061 \\
\hline $\mathrm{H}(2 \mathrm{~B})$ & $4 e$ & 0.5703 & 0.2036 & 0.2086 & 0.061 \\
\hline $\mathrm{H}(2 \mathrm{C})$ & $4 e$ & 0.6054 & 0.2009 & 0.3423 & 0.061 \\
\hline $\mathrm{H}(3 \mathrm{~A})$ & $4 e$ & 0.6519 & 0.0271 & 0.3684 & 0.037 \\
\hline $\mathrm{H}(3 \mathrm{~B})$ & $4 e$ & 0.6838 & 0.1020 & 0.3635 & 0.037 \\
\hline $\mathrm{H}(4 \mathrm{~A})$ & $4 e$ & 0.6708 & 0.0766 & 0.5415 & 0.059 \\
\hline $\mathrm{H}(4 \mathrm{~B})$ & $4 e$ & 0.5378 & 0.0525 & 0.4868 & 0.059 \\
\hline $\mathrm{H}(4 \mathrm{C})$ & $4 e$ & 0.5697 & 0.1276 & 0.4819 & 0.059 \\
\hline $\mathrm{H}(5 \mathrm{~A})$ & $4 e$ & 0.5978 & 0.0302 & 0.1673 & 0.037 \\
\hline $\mathrm{H}(5 \mathrm{~B})$ & $4 e$ & 0.6209 & 0.1065 & 0.1688 & 0.037 \\
\hline $\mathrm{H}(6 \mathrm{~A})$ & $4 e$ & 0.5030 & $0.0 \overline{742}$ & -0.0109 & 0.056 \\
\hline $\mathrm{H}(6 \mathrm{~B})$ & $4 e$ & 0.4290 & 0.1223 & 0.0415 & 0.056 \\
\hline $\mathrm{H}(6 \mathrm{C})$ & $4 e$ & 0.4058 & 0.0458 & 0.0401 & 0.056 \\
\hline $\mathrm{H}(7 \mathrm{~A})$ & $4 e$ & 0.3919 & 0.0371 & 0.3263 & 0.035 \\
\hline $\mathrm{H}(7 \mathrm{~B})$ & $4 e$ & 0.3515 & 0.0377 & 0.1925 & 0.035 \\
\hline $\mathrm{H}(8 \mathrm{~A})$ & $4 e$ & 0.3919 & -0.0689 & 0.2583 & 0.059 \\
\hline $\mathrm{H}(8 \mathrm{~B})$ & $4 e$ & 0.5237 & -0.0489 & 0.3286 & 0.059 \\
\hline $\mathrm{H}(8 \mathrm{C})$ & $4 e$ & 0.4833 & -0.0483 & 0.1945 & 0.059 \\
\hline $\mathrm{H}(9 \mathrm{~A})$ & $4 e$ & -0.0090 & -0.1565 & 0.3357 & 0.041 \\
\hline $\mathrm{H}(9 \mathrm{~B})$ & $4 e$ & 0.1081 & -0.1215 & 0.3294 & 0.041 \\
\hline $\mathrm{H}(10 \AA)$ & $4 e$ & 0.0916 & -0.1102 & 0.5095 & 0.065 \\
\hline $\mathrm{H}(10 \mathrm{~B})$ & $4 e$ & -0.0334 & -0.0752 & 0.4591 & 0.065 \\
\hline $\mathrm{H}(10 \mathrm{C})$ & $4 e$ & 0.0840 & -0.0402 & 0.4529 & 0.065 \\
\hline $\mathrm{H}(11 \mathrm{~A})$ & $4 e$ & -0.1685 & -0.0404 & 0.3030 & 0.041 \\
\hline$H(11 B)$ & $4 e$ & -0.2131 & -0.0388 & 0.1691 & 0.041 \\
\hline $\mathrm{H}(12 \mathrm{~A})$ & $4 e$ & -0.3195 & -0.1166 & 0.2340 & 0.074 \\
\hline $\mathrm{H}(12 \mathrm{~B})$ & $4 e$ & -0.2001 & -0.1537 & 0.2991 & 0.074 \\
\hline $\mathrm{H}(12 \mathrm{C})$ & $4 e$ & -0.2448 & -0.1520 & 0.1650 & 0.074 \\
\hline $\mathrm{H}(13 \mathrm{~A})$ & $4 e$ & -0.0423 & 0.0160 & 0.1651 & 0.036 \\
\hline$H(13 B)$ & $4 e$ & 0.0023 & 0.0169 & 0.2990 & 0.036 \\
\hline $\mathrm{H}(14 \mathrm{~A})$ & $4 e$ & 0.1599 & 0.0394 & 0.2307 & 0.065 \\
\hline $\mathrm{H}(14 \mathrm{~B})$ & $4 e$ & 0.1403 & -0.0282 & 0.1649 & 0.065 \\
\hline $\mathrm{H}(14 \mathrm{C})$ & $4 e$ & 0.1850 & -0.0273 & 0.2990 & 0.065 \\
\hline $\mathrm{H}(15 \mathrm{~A})$ & $4 e$ & 0.0422 & -0.1201 & 0.1341 & 0.039 \\
\hline $\mathrm{H}(15 \mathrm{~B})$ & $4 e$ & -0.0743 & -0.1577 & 0.1351 & 0.039 \\
\hline $\mathrm{H}(16 \mathrm{~A})$ & $4 e$ & -0.1004 & -0.1130 & -0.0420 & 0.059 \\
\hline
\end{tabular}


Table 2. Continued.

\begin{tabular}{llllll}
\hline Atom & Site & $x$ & $y$ & $z$ & $U_{\text {iso }}$ \\
\hline $\mathrm{H}(16 \mathrm{~B})$ & $4 e$ & -0.0738 & -0.0420 & 0.0114 & 0.059 \\
$\mathrm{H}(16 \mathrm{C})$ & $4 e$ & -0.1907 & -0.0797 & 0.0124 & 0.059 \\
$\mathrm{H}(17 \mathrm{~A})$ & $4 e$ & 0.4581 & -0.3521 & 0.1675 & 0.033 \\
$\mathrm{H}(17 \mathrm{~B})$ & $4 e$ & 0.5045 & -0.3514 & 0.3013 & 0.033 \\
$\mathrm{H}(18 \mathrm{~A})$ & $4 e$ & 0.6606 & -0.3759 & 0.2314 & 0.060 \\
$\mathrm{H}(18 \mathrm{~B})$ & $4 e$ & 0.6871 & -0.3084 & 0.2973 & 0.060 \\
$\mathrm{H}(18 \mathrm{C})$ & $4 e$ & 0.6406 & -0.3091 & 0.1632 & 0.060 \\
$\mathrm{H}(19 \mathrm{~A})$ & $4 e$ & 0.2886 & -0.2962 & 0.1688 & 0.033 \\
$\mathrm{H}(19 \mathrm{~B})$ & $4 e$ & 0.3322 & -0.2928 & 0.3026 & 0.033 \\
$\mathrm{H}(20 \mathrm{~A})$ & $4 e$ & 0.1832 & -0.2157 & 0.2295 & 0.057 \\
$\mathrm{H}(20 \mathrm{~B})$ & $4 e$ & 0.2597 & -0.1825 & 0.1600 & 0.057 \\
& & & & & \\
\hline
\end{tabular}

Table 2. Continued.

\begin{tabular}{llllll}
\hline Atom & Site & $x$ & $y$ & \multicolumn{1}{l}{$z$} & $U_{\text {iso }}$ \\
\hline $\mathrm{H}(20 \mathrm{C})$ & $4 e$ & 0.3034 & -0.1790 & 0.2940 & 0.057 \\
$\mathrm{H}(21 \mathrm{~A})$ & $4 e$ & 0.4318 & -0.1779 & 0.1341 & 0.036 \\
$\mathrm{H}(21 \mathrm{~B})$ & $4 e$ & 0.5458 & -0.2178 & 0.1324 & 0.036 \\
$\mathrm{H}(22 \mathrm{~A})$ & $4 e$ & 0.4010 & -0.2242 & -0.0432 & 0.058 \\
$\mathrm{H}(22 \mathrm{~B})$ & $4 e$ & 0.3099 & -0.2551 & 0.0129 & 0.058 \\
$\mathrm{H}(22 \mathrm{C})$ & $4 e$ & 0.4242 & -0.2951 & 0.0112 & 0.058 \\
$\mathrm{H}(23 \mathrm{~A})$ & $4 e$ & 0.6118 & -0.2166 & 0.3318 & 0.035 \\
$\mathrm{H}(23 \mathrm{~B})$ & $4 e$ & 0.4963 & -0.1776 & 0.3315 & 0.035 \\
$\mathrm{H}(24 \mathrm{~A})$ & $4 e$ & 0.5861 & -0.2227 & 0.5093 & 0.056 \\
$\mathrm{H}(24 \mathrm{~B})$ & $4 e$ & 0.5746 & -0.2938 & 0.4560 & 0.056 \\
$\mathrm{H}(24 \mathrm{C})$ & $4 e$ & 0.4588 & -0.2547 & 0.4558 & 0.056 \\
\hline
\end{tabular}

Table 3. Atomic coordinates and displacement parameters (in $\AA^{2}$ ).

\begin{tabular}{|c|c|c|c|c|c|c|c|c|c|c|}
\hline Atom & Site & $x$ & $y$ & $z$ & $U_{11}$ & $U_{22}$ & $U_{33}$ & $U_{12}$ & $U_{13}$ & $U_{23}$ \\
\hline $\mathrm{Cl}(1)$ & $4 e$ & $0.22881(5)$ & $-0.08198(3)$ & $-0.00897(4)$ & $0.0451(3)$ & $0.0399(3)$ & $0.0360(3)$ & $0.0085(2)$ & $0.0100(2)$ & $0.0026(2)$ \\
\hline $\mathrm{Cl}(2)$ & $4 e$ & $0.79225(5)$ & $0.25494(3)$ & $0.51659(4)$ & $0.0406(3)$ & $0.0457(3)$ & $0.0322(3)$ & $-0.0036(2)$ & $0.0085(2)$ & $-0.0009(2)$ \\
\hline $\mathrm{Cl}(3)$ & $4 e$ & $0.26751(5)$ & $0.08822(3)$ & $0.48435(5)$ & $0.0478(3)$ & $0.0429(3)$ & $0.0398(3)$ & $0.0120(3)$ & $0.0087(2)$ & $0.0010(2)$ \\
\hline$N(1)$ & $4 e$ & $0.5144(1)$ & $0.08033(7)$ & $0.2638(1)$ & $0.0211(7)$ & $0.0229(8)$ & $0.0228(7)$ & $-0.0004(6)$ & $0.0061(6)$ & $0.0013(6)$ \\
\hline $\mathrm{N}(2)$ & $4 e$ & $-0.0439(1)$ & $-0.07506(7)$ & $0.2339(1)$ & $0.0255(8)$ & $0.0230(8)$ & $0.0250(8)$ & $0.0029(6)$ & $0.0074(6)$ & $0.0008(6)$ \\
\hline$C(1)$ & $4 e$ & $0.4572(2)$ & $0.14670(9)$ & $0.2639(2)$ & $0.030(1)$ & $0.026(1)$ & $0.029(1)$ & $0.0042(8)$ & $0.0071(8)$ & $0.0008(8)$ \\
\hline$C(2)$ & $4 e$ & $0.5397(2)$ & $0.2040(1)$ & $0.2724(2)$ & $0.049(1)$ & $0.027(1)$ & $0.042(1)$ & $-0.0049(9)$ & $0.008(1)$ & $0.0009(9)$ \\
\hline $\mathrm{C}(3)$ & $4 e$ & $0.6213(2)$ & $0.0717(1)$ & $0.3684(2)$ & $0.0234(9)$ & $0.037(1)$ & $0.028(1)$ & $0.0015(8)$ & $0.0026(7)$ & $0.0027(8)$ \\
\hline $\mathrm{C}(4)$ & $4 e$ & $0.5978(2)$ & $0.0831(1)$ & $0.4796(2)$ & $0.042(1)$ & $0.048(1)$ & $0.024(1)$ & $0.002(1)$ & $0.0059(9)$ & $0.0028(9)$ \\
\hline$C(5)$ & $4 e$ & $0.5596(2)$ & $0.0732(1)$ & $0.1630(2)$ & $0.031(1)$ & $0.037(1)$ & $0.028(1)$ & $0.0023(8)$ & $0.0144(8)$ & $-0.0002(8)$ \\
\hline$C(6)$ & $4 e$ & $0.4659(2)$ & $0.0795(1)$ & $0.0481(2)$ & $0.041(1)$ & $0.046(1)$ & $0.025(1)$ & $0.003(1)$ & $0.0114(9)$ & $-0.0004(9)$ \\
\hline$C(7)$ & $4 e$ & $0.4194(2)$ & $0.03013(9)$ & $0.2605(2)$ & $0.027(1)$ & $0.028(1)$ & $0.031(1)$ & $-0.0047(8)$ & $0.0081(8)$ & $-0.0001(8)$ \\
\hline$C(8)$ & $4 e$ & $0.4580(2)$ & $-0.0403(1)$ & $0.2605(2)$ & $0.051(1)$ & $0.027(1)$ & $0.039(1)$ & $-0.0033(9)$ & $0.012(1)$ & $-0.0001(9)$ \\
\hline C (9) & $4 e$ & $0.0289(2)$ & $-0.1137(1)$ & $0.3366(2)$ & $0.041(1)$ & $0.029(1)$ & $0.029(1)$ & $0.0082(9)$ & $0.0070(9)$ & $0.0055(8)$ \\
\hline$C(10)$ & $4 e$ & $0.0441(2)$ & $-0.0820(1)$ & $0.4496(2)$ & $0.055(1)$ & $0.044(1)$ & $0.027(1)$ & $0.003(1)$ & $0.009(1)$ & $0.001(1)$ \\
\hline$C(11)$ & $4 e$ & $-0.1705(2)$ & $-0.0654(1)$ & $0.2350(2)$ & $0.027(1)$ & $0.040(1)$ & $0.036(1)$ & $-0.0001(9)$ & $0.0118(8)$ & $-0.0048(9)$ \\
\hline$C(12)$ & $4 e$ & $-0.2399(2)$ & $-0.1274(1)$ & $0.2331(2)$ & $0.043(1)$ & $0.058(2)$ & $0.048(1)$ & $-0.022(1)$ & $0.017(1)$ & $-0.006(1)$ \\
\hline$C(13)$ & $4 e$ & $0.0073(2)$ & $-0.00718(9)$ & $0.2324(2)$ & $0.031(1)$ & $0.025(1)$ & $0.032(1)$ & $-0.0025(8)$ & $0.0083(8)$ & $0.0015(8)$ \\
\hline$C(14)$ & $4 e$ & $0.1344(2)$ & $-0.0057(1)$ & $0.2317(2)$ & $0.031(1)$ & $0.060(2)$ & $0.038(1)$ & $-0.010(1)$ & $0.0091(9)$ & $0.005(1)$ \\
\hline$C(15)$ & $4 e$ & $-0.0411(2)$ & $-0.1142(1)$ & $0.1307(2)$ & $0.039(1)$ & $0.030(1)$ & $0.029(1)$ & $0.0079(8)$ & $0.0102(9)$ & $-0.0033(8)$ \\
\hline$C(16)$ & $4 e$ & $-0.1074(2)$ & $-0.0846(1)$ & $0.0182(2)$ & $0.050(1)$ & $0.039(1)$ & $0.027(1)$ & $0.004(1)$ & $0.0088(9)$ & $-0.0002(9)$ \\
\hline$C(17)$ & $4 e$ & $0.5086(2)$ & $-0.32834(9)$ & $0.2335(2)$ & $0.031(1)$ & $0.0236(9)$ & $0.0277(9)$ & $0.0049(8)$ & $0.0087(8)$ & $0.0004(8)$ \\
\hline$C(18)$ & $4 e$ & $0.6356(2)$ & $-0.3307(1)$ & $0.2311(2)$ & $0.032(1)$ & $0.052(1)$ & $0.035(1)$ & $0.010(1)$ & $0.0094(9)$ & $-0.002(1)$ \\
\hline$C(19)$ & $4 e$ & $0.3312(2)$ & $-0.26900(9)$ & $0.2338(2)$ & $0.0232(9)$ & $0.031(1)$ & $0.028(1)$ & $-0.0013(7)$ & $0.0081(7)$ & $0.0014(8)$ \\
\hline$C(20)$ & $4 e$ & $0.2633(2)$ & $-0.2059(1)$ & $0.2289(2)$ & $0.034(1)$ & $0.042(1)$ & $0.041(1)$ & $0.0107(9)$ & $0.0139(9)$ & $0.004(1)$ \\
\hline$C(21)$ & $4 e$ & $0.4625(2)$ & $-0.2221(1)$ & $0.1298(2)$ & $0.033(1)$ & $0.030(1)$ & $0.029(1)$ & $-0.0035(8)$ & $0.0105(8)$ & $0.0063(8)$ \\
\hline$C(22)$ & $4 e$ & $0.3932(2)$ & $-0.2518(1)$ & $0.0177(2)$ & $0.046(1)$ & $0.046(1)$ & $0.025(1)$ & $0.001(1)$ & $0.0097(9)$ & $0.0057(9)$ \\
\hline$C(23)$ & $4 e$ & $0.5308(2)$ & $-0.2215(1)$ & $0.3358(2)$ & $0.030(1)$ & $0.028(1)$ & $0.030(1)$ & $-0.0055(8)$ & $0.0066(8)$ & $-0.0039(8)$ \\
\hline$C(24)$ & $4 e$ & $0.5382(2)$ & $-0.2508(1)$ & $0.4493(2)$ & $0.042(1)$ & $0.043(1)$ & $0.024(1)$ & $-0.002(1)$ & $0.0058(9)$ & $-0.0033(9)$ \\
\hline
\end{tabular}

\section{References}

1. Loehlin, J. H.; Kvick, A.: Tetraethylammonium Chloride Monohydrate. Acta Crystallogr. B34 (1978) 3488-3490.

2. Mak, T. C. W.; Slot, H. J. B.; Beurskens, P. T.: Tetraethylammonium Chloride Tetrahydrate. J. Inclusion Phenom. 4 (1986) 295-298.
3. Sheldrick, G. M.: Phase Annealing in SHELX-90: Direct Methods for Large Structures. Acta Crystallogr. A46 (1990) 467-473.

4. Sheldrick, G. M.; SHELXL-97. Program for refining crystals structures. University of Göttingen, Germany 1997. 